\title{
Hostility and the Progression of Carotid Atherosclerosis
}

\author{
Juhani Julkunen, Phlic, Rittta Salonen, MD, PhD, George A. Kaplan, PhD, \\ Margaret A. Chesney, PhD, and Jukka T. Salonen, MD, PhD, MPH
}

\begin{abstract}
We studied prospectively the association of hostility and anger suppression by the use of ultrasonographically assessed 2-year progression of carotid atherosclerosis (PCA) in a sample of 119 middle-aged men from eastern Finland. Based on measures of cynical distrust, impatience-irritability, anger-in, and anger-control, four variants of the hostility-by-anger suppression model were tested with multiple regression analysis. In addition to the previously established risk factors (i.e., serum low-density lipoprotein cholesterol concentration, smoking, and old age), cynical distrust and anger-control significantly predicted PCA. There was about a two-fold accelerated PCA in the group with high cynical distrust and high anger-control even after we controlled for the established biological risk factors and possible confounding background variables. The impact of the independent variables on PCA seemed to be additive rather than synergistic. These results, based on a relatively small, but nonselected population sample, extend previous results of angiographic studies.

Key words: anger, atherosclerosis, hostility, ultrasonography.
\end{abstract}

\section{INTRODUCTION}

Our current knowledge of the risk factors for atherosclerosis is largely based on angiographic studies limited to selected clinical samples. The methodological problems of angiographic studies have been critically discussed, and it has been argued that they are not well suited to the investigation of behavioral risk factors (1). On the other hand, advances in ultrasonographic techniques enable us to study the progression of extracoronary atherosclerosis in nonclinical population samples (2). Furthermore, recent data $(2,3)$ suggest that ultrasonographically assessed common carotid atherosclerosis has a strong association with the risk of coronary heart disease (CHD). Also, human autopsy studies have established a close association between carotid and coronary atherosclerosis (for a review, see Ref. 2). Thus, studies of the risk factors for extracoronary atherosclerosis offer a potentially important method for epidemiological research into the risk factors for coronary atherosclerosis and CHD.

Substantial cross-sectional and prospective epi-

From the Rehabilitation Foundation, Helsinki (J.J.), and the Research Institute of Public Health, University of Kuopio (R S. J.T.S.), Kuopio, Finland; and the Human Population Laboratory California Department of Health Services, Berkeley (G.A.K.), and the Prevention Sciences Center, University of California, San Francisco (M.A.C.), Californıa.

Address reprint requests to: Professor Jukka T. Salonen, Professor of the Academy of Finland, Research Institute of Public Health, University of Kuopio, P.O. Box 1627, 70211 Kuopio, Finland.

Received for publication June 18, 1992; revision received April 21, 1994 demiological research indicates that hostility and anger may confer an increased risk of coronary atherosclerosis and CHD $(4,5)$. This conclusion is supported by a meta-analysis by Matthews (6) and by a recent literature review (7). As a psychological construct, hostility is associated with a large number of phenomena, i.e., aggression, anger, annoyance, irritability, suspicion, and cynicism. It has been proposed that three aspects of hostility should be separated as concepts and targets for assessment (8). The cognitive component of hostility consists of negative beliefs of others who are seen as unreliable, undeserving, immoral, and possibly threatening antagonists. The affective component includes several emotional states, such as anger, irritability, disgust, and contempt. The third component covers the various forms of aggressive behavior from sulking and verbal aggression to overtly aggressive acts (8).

From this perspective, we might ask whether all these three aspects of hostility are equally important as risk factors for CHD and, furthermore, whether various combinations of these components have different implications for health. Although several authors have underlined the complex nature of hostil. ity and aggressive behavior (8-10), research practice has often favored single-factor, univariate analysis. An exception to this trend is the study by Dembroski et al. (11), in which several aspects of hostility and Type A behavior were investigated in relationship to angiographic findings. The results indicated that the potential for hostility was significantly associated with the extent of coronary atherosclerosis but only for patients who were high on anger-in (AXin). The authors argue that hostility in conjunction with acquired coping mechanisms, such as anger 


\section{J. JULKUNEN et al.}

suppression (AX-in), might be the core of coronaryprone behavior. Although McDougall et al. (12) did not replicate the findings of Dembroski et al. (11), Suarez (13), using a sample of 277 patients with coronary artery disease, found a significant interaction between the potential for hostility and AX-in whereby highly hostile men who suppressed their anger evidenced more severe disease.

So far, the conclusions of these studies are limited to selected clinical samples. When we consider the interesting results, surprisingly little research has been done to replicate or test these kinds of interactive models in prospective studies based on nonclinical samples.

The aim of the present study was to investigate the cognitive and affective components of hostility in combination with anger suppression as possible predictors of 2-year progression of carotid atherosclerosis (PCA), as measured ultrasonographically. We hypothesized that both components of hostility would be associated with PCA. In addition, in accordance with the results of Dembroski et al. (11) and Suarez (13), we expected a synergistic interaction effect between hostility and anger suppression in the prediction of PCA.

\section{METHODS}

\section{Subjects}

The Kuopio Ischaemic Heart Disease Risk Factor Study (KIHD) is a population study to investigate previously unestablished risk factors for CHD and extracoronary atherosclerosis (14). The study population consists of a stratified random $33 \%$ sample $(N=2,682)$ of men aged either $42,48,54$, or 60 years at the time of baseline measurements, who were living in a geographically defined area in eastern Finland. The design and methods for the main study have been described in detail elsewhere (14).

The present study sample consisted of a subsample drawn from the population sample of the KIHD study. The subjects of the present study were KIHD participants who were examined between February and May and between October and November 1987. The periods of subject entry were chosen on the basis of study logistics. A total of 128 men ( $93 \%$ of those eligible) participated in a 2-year ultrasonographic reexamination; 119 men answered the psychological questionnaire. The mean age of the subjects was 54 years (SD, 5.9). The subjects did not differ from all KIHD participants in either socioeconomic characteristics or the variables studied in this article.

\section{Assessment of Atherosclerosis}

A high-resolution B-mode ultrasonographic examination was repeated after a follow-up of approximately 24 months for each subject. The site of the most advanced atherosclerotic lesion and the projection that showed the greatest distance between the lumen-intimal interface and the media-adventitia interface (intıma-media thickness, IMT) was located in both the right and left common carotid artery. The scanning procedure was recorded on a video cassette recorder. The IMT was measured from these recordings by the scanning physician (R.S.), who was blind to the subject's risk factor values. The reading of the follow-up recording was blind with regard to baseline IMT values. Three measurements of IMT were carried out on the far wall of both the right and left common carotid artery at the site of the greatest IMT each time. The mean of these six IMT measurements was used to estimate IMT each time. The difference between the 2-year mean IMT and the baseline mean IMT was used as the indicator of PCA. A more detailed description of the procedure of the ultrasonographic measurements and other medical baseline measurements has been reported elsewhere $(2,14-16)$. In a blinded reproducibility study by the same observer in 41 subjects, the Pearson's correlation coefficients for the repeat scannings were $.98, .99$ for repeat IMT measurements, and .97 for the entire assessment of IMT (17). On the basis of a phantom experiment and reproducibility studies, the measurement precision was of the order of 0.03 to $0.08 \mathrm{~mm}(17,18)$.

\section{Assessment of Psychological Risk Factors}

The psychological data used in this study were collected by a self-report questionnaire mailed to the participants approximately in the middle of the 2-year follow-up period. Because of the relatively high 12 -month retest reliabilities for the measures used in this study (Table 1), the collection of these data after baseline was not thought to cause any substantial problems for the prospective study.

Considering the complexity of the hostility construct, we assessed the cognitive and affective components with separate measures. In addition, two measures were used to assess anger suppression as a variant of the behavioral component of hostility.

The measure for the cognitive component of hostility, the Cynical Distrust scale (CynDis), was factor-analytically derived from the Cook-Medley hostility scale (19) by Greenglass and Julkunen (20). The results of previous studies indicated the CynDis scale to be a reliable, valid, and more specific measure of cynicism and distrust compared with the total Cook-Medley scale $(20,21)$. In two separate samples of Finnish and Canadian students, the scale correlated significantly with the total CookMedley hostility scale $(r=.77, p<.001)$. Moreover, in these samples CynDis correlated higher with other measures of cynicism and distrust than with anger-out or irritability; the total hostility scale correlated substantially also with these measures $(20,21)$. Illustrative items of the scale are, e.g., "No one cares much what happens to you" and "It is safer to trust nobody." In the present study, we used the eight items with the highest factor loadings on the first factor, as indicated in the previous study (20). Instead of the original dichotomous response form, we used

TABLE 1. Number of Items, Internal Consistency and TestRetest Reliability of the Psychological Measures

\begin{tabular}{lccc}
\hline & $\begin{array}{c}\text { Numbers } \\
\text { of Items }\end{array}$ & $\begin{array}{c}\text { Cronbach's } \\
\text { Alpha }\end{array}$ & $\begin{array}{c}\text { Retest } \\
\text { Reliability }(r)^{\mathrm{a}}\end{array}$ \\
\hline Cynical distrust & $\mathbf{8}$ & 0.81 & 0.82 \\
Impatience-irritability & $\mathbf{8}$ & 0.64 & 0.72 \\
Anger-in & 8 & 0.70 & 0.66 \\
Anger-control & 8 & 0.88 & 0.76 \\
\hline
\end{tabular}




\section{HOSTILITY AND ATHEROSCLEROSIS}

a four-step format to answer the questions ("completely agree," "somewhat agree," "somewhat disagree," and "completely disagree"). In this way, greater variance and a nearly normal distribution of the test score were obtained.

The measure for the affective component of the hostility was the Impatience and Irritability factor (FTA-I) derived from The Finnish Type A scale (22). The eight-item subscale primarily reflects the hurried life-style component of the Type A construct and also includes some assessment of irritability Representative items are, e.g., "I am easygoing by nature" (not true) and "My attitude toward life is casual, and I am not easily irritated" (not true).

Suppression of anger was measured with two subscales from the 24-item Anger Expression Scale developed by Spielberger et al. $(9,23) . A X$-in refers to a tendency to hold in or suppress angry feelings. Representative items are "I boil inside, but I don't show it" and "I am angrier than I am willing to admit." Anger-control (AX-con) is characterized by efforts to control angry feelings and reactions by avoiding open expression of irritation or aggression. Representative items are "I control my behavior" and "I try to be tolerant and understanding." Romanov et al. (24) studied the validity of the Anger Expression subscales in a sample of 550 Finnish men and women. They found relatively good/satisfactory agreement between spouse's assessment and the self-report scores on the Anger Expression subscales. For the scales used in this study, the Pearson correlation coefficients between spouse's assessment and self-report were .39 for both men and women on the $\mathrm{AX}$-in and, on the AX-con, .43 for men and .34 for women. Also the alpha coefficients for the internal consistency of these scales were satisfactory and corresponded well to the previous results in the original samples from the United States. A more detailed description about the validity and psychometric properties of the anger expression scales is available in the State-Trait Anger Inventory manual (23).

Based on these measures, four hypothetical hostility-anger suppression models for the prediction of PCA were formed as follows:

1. CynDis and $A X-i n$,

2. FTA-I and $A X$-in,

3. CynDis and $A X-c o n$, and

4. FTA-I and AX-con.

Reliability data for the measures used in this study are presented in Table 1. Cronbach's alpha coefficients for internal consistency were based on the entire KIHD sample, and the test-retest (12 months) correlations were obtained from a random subsample ( $N$ $=50$ ) of the whole KIHD sample. Socioeconomic status (SES) was measured with a summary index that combined measures of income, education, occupation, occupational prestige, material standard of living, and housing conditions (25).

\section{Statistical Analyses}

Pearson's correlation coefficients were used to estimate the independence of the psychological measures and their associations with the social and biological background variables. Multivariate regression analyses were applied to test the four proposed models as predictors of the 2-year PCA. Regression analyses were performed with both continuous and binary predictors. Two-way analyses of covariance (ANCOVA) were used to illustrate the predictive power of classified independent variables and their interactions in the prediction of PCA.

In previous analyses $(2,16,26)$, age, cigarette-years, and serum low-density lipoprotein (LDL) cholesterol concentration were the strongest predictors of PCA. These were forced into all multivariate models. Other forced covariates were the SES, baseline common carotid IMT, month and season of baseline examination, and exact follow-up time in days. Because neither blood pressure, serum high-density lipoprotein cholesterol, nor family history of CHD had an association with PCA in the previous analyses $(2,16$, $26)$, these were not entered as covariates.

Because the dependent variable, 2-year change in IMT, was skewed to the right, a log-transformation was used in all multivariate analyses (a constant value of 0.07 was first added to transform negative values to positive).

To create the sum indexes for the psychological measures, missing items were replaced by personal mean values based on items to which responses were made. This was done in four cases for CynDis, three cases for FTA-I, four for AX-con, and one for $\mathrm{AX}$-in variable. If one half or more of the items was missing, the sum index was defined as missing. In multivariate analyses, the missing values (one for $\mathrm{AX}$-con and three for $\mathrm{AX}$-in) were replaced by grand means.

The SPSS-X software (27) for IBM RS/6000 computers (International Business Machines, ) was used in all statistical analysis.

\section{RESULTS}

The mean 2-year increase of the ultrasonographically assessed common carotid IMT in this sample was $0.11 \mathrm{~mm}$ (SD, $0.19 \mathrm{~mm}$; range, $0.06-0.90 \mathrm{~mm}$ ).

Intercorrelations of the psychological measures (Table 2) indicated that CynDis and FTA-I had a low and insignificant correlation, which thus apparently reflected different aspects of general hostility. The same was true with the measures that assessed anger suppression, which indicated that $\mathrm{AX}$-con and AXin did not assess the same type of coping with anger. FTA-I and AX-con had a strong negative correlation.

Zero-order correlational analysis, summarized in Table 3, showed that CynDis was significantly associated with PCA $(r=.24, p=.008)$ and with the baseline IMT ( $r=.21, p=.024)$. CynDis also correlated positively with old age and with low SES. The $\mathrm{AX}$-con correlated almost significantly $(\mathrm{r}=.17, \mathrm{p}=$ .07) with PCA. Other correlations were negligible.

The four variants of the proposed hostility-anger suppression model that predict PCA were tested with multivariate regression analyses by both con-

TABLE 2. Correlations of the Psychological Measures

\begin{tabular}{lccc}
\hline & $\begin{array}{c}\text { Cynial } \\
\text { Distrust }\end{array}$ & $\begin{array}{c}\text { Impatience- } \\
\text { Irritabılity }\end{array}$ & $\begin{array}{c}\text { Anger- } \\
\text { Control }\end{array}$ \\
\hline Anger-in & $0.20^{\star}$ & 0.12 & -0.08 \\
Anger-control & -0.07 & $-0.55^{\star \star}$ & \\
Impatience-irritability & 013 & & \\
\hline
\end{tabular}

$N=119$.

${ }^{*} p<0.05$.

$\star p<0.001$ 


\section{J. JULKUNEN et al.}

tinuous and dichotomous predictors. The results are summarized in Table 4 . We also entered the interactions into all the presented models, but this calculation did not raise the $\mathrm{R}^{2}$ in any of the models. For this reason, models that include interaction terms are not presented.

Two of the hypothesized predictive variables, CynDis and $A X$-con, showed consistently significant residual associations with $\mathrm{PCA}$; in Model 1, the main effect of the continuous CynDis was just short of statistical significance $(p=.07)$. At the same time, the other variables, FTA-I and AX-in, did not have any statistically significant association with PCA in any of the models.

Model 3, based on dichotomous CynDis and AXcon, was the best to account for the variability of the 2-year PCA $\left(\mathrm{R}^{2}=.37\right)$. To illustrate this model a $2 \times 2$ ANCOVA was performed that controlled for the same covariates as used in the regression analyses and used the same cut points for the binary predictors. The adjusted mean values are presented in Figure 1. The results indicate a two-fold accelerated PCA in the group that was high in both predictors compared with the low-exposure group. The nontransformed mean values indicated more than five-fold greater PCA in the high-exposure group compared with the group below the median on both predictors $(0.03 \mathrm{~mm}$ vs. $0.17 \mathrm{~mm}$, adjusted values, respectively).

\section{DISCUSSION}

In the present study, we investigated the role of two components of hostility together with two indicators of anger suppression as possible predictors of PCA during a 2-year follow-up of a random population sample. We expected both the cognitive and the affective components (as indicated by CynDis and FTA-I, respectively) to be associated with PCA. For CynDis, the hypothesis was supported consistently. For the FTA-I, the hypothesis was not supported by the present results.

These findings suggest that the cognitive component of hostility measured by the CynDis scale is more important as a risk factor for atherosclerosis than the affective component measured by the FTAI scale. This conclusion, however, must be taken cautiously. First, although relatively good reliability was shown, the FTA-I was less reliable (Table 1) and not as well validated a measure as CynDis. Furthermore, in other analyses (not reported here), when the direct, not log-transformed values of PCA were used as the dependent variable, FTA-I actually had

TABLE 3. Crude Correlation Coefficients for the Associations of the Psychological Measures With the Progression of Carotid Atherosclerosis, Baseline IMT, Age, Serum LDL Cholesterol (LDL), Cigarette-Years, and Low Socioeconomic Status

\begin{tabular}{|c|c|c|c|c|c|c|}
\hline Varıable & $\begin{array}{c}\text { Progression of Carotid } \\
\text { Atherosclerosis }\end{array}$ & $\begin{array}{l}\text { Baseline } \\
\text { IMT }\end{array}$ & Age & $\mathrm{LDL}$ & $\begin{array}{l}\text { Cigarette- } \\
\text { years }\end{array}$ & $\begin{array}{c}\text { Low Socioeconomic } \\
\text { Status }\end{array}$ \\
\hline Cynical distrust & $0.24^{* *}$ & $0.21^{\star}$ & $0.23^{*}$ & -0.03 & 0.15 & $0.27^{\star \star}$ \\
\hline Impatience-irritability & 0.08 & 0.03 & -0.08 & 0.07 & 0.01 & -0.08 \\
\hline Anger-in & 0.07 & 0.00 & 0.07 & -0.18 & 0.10 & 0.15 \\
\hline Anger-control & 0.17 & 0.10 & 0.12 & 0.06 & -0.06 & -0.05 \\
\hline
\end{tabular}

${ }^{*} p<0.05$

$* p<0.01$.

TABLE 4. Summary of the Four Multivariate Regression Models Based on Continuous (cont) and Dichotomized (bin) Variables as Predictors of 2-Year Progression of Carotid Atherosclerosis

\begin{tabular}{|c|c|c|c|c|c|c|c|c|}
\hline & \multicolumn{8}{|c|}{ Standardized Regression Coefficient } \\
\hline & \multicolumn{2}{|c|}{ Model 1} & \multicolumn{2}{|c|}{ Model 2} & \multicolumn{2}{|c|}{ Model 3} & \multicolumn{2}{|c|}{ Model 4} \\
\hline & Contınuous & Binary & Continuous & Binary & Continuous & Bınary & Continuous & Binary \\
\hline Cynical distrust & 0.16 & $0.26^{\star *}$ & & & $0.17^{\star}$ & $0.27^{\star \star \star}$ & & \\
\hline Impatience-irritability & & & 0.01 & -0.04 & & & 0.14 & 0.05 \\
\hline Anger-in & -0.01 & 0.05 & 0.02 & 0.07 & & & & \\
\hline Anger-control & & & & & $0.16^{*}$ & $0.18^{*}$ & $0.23^{*}$ & $0.20^{\star}$ \\
\hline$R^{2}$ & $0.30^{\star \star *}$ & $0.35^{\star \star \star}$ & $0.28^{\star \star *}$ & $0.28^{\star \star \star}$ & $0.32^{\star \star *}$ & $0.37^{\star \star \star}$ & 0.31 th & $0.31^{\text {nt }}$ \\
\hline
\end{tabular}

Note: All regression models included age (years, $p<0.05)$, cigarette-years $(p<0.001)$, serum LDL cholesterol concentration $(p<0.05)$, SES (NS), baseline IMT (NS), month (NS) and season of baseline examination (NS), and length of follow-up time (days, NS).

${ }^{\star} p<0.05 ;{ }^{* \star} p<0.01 ;{ }^{* \star \star} p<0.001$. 


\section{HOSTILITY AND ATHEROSCLEROSIS}

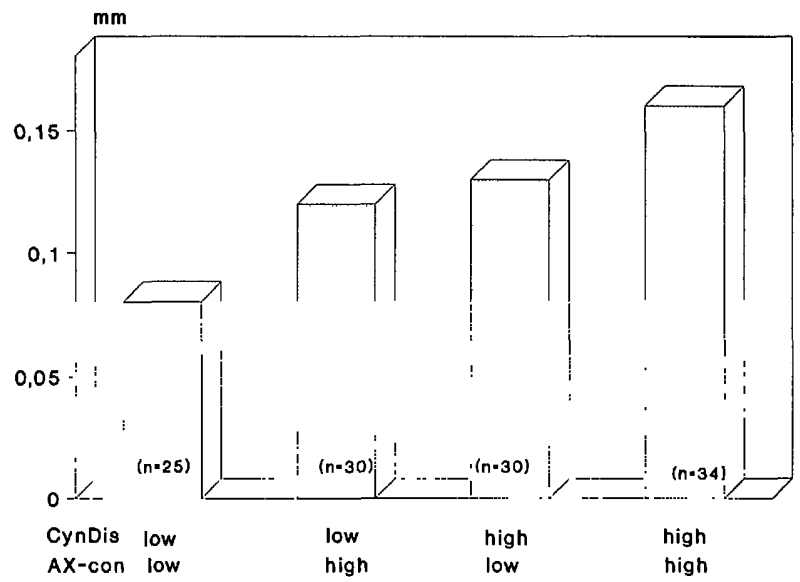

Fig. 1. Two-year PCA by CynDis and AX-con adjusted for age, cigarette-years, serum LDL cholesterol, SES status, baseline carotid atherosclerosis, season of baseline examination, and days of follow-up.

a significant ( $p=.01$ ) association with PCA in Model 4. The role of the affective components of hostility as a risk factor for PCA deserves further study with well-established measures and larger samples.

The results based on Model 3, including CynDis and AX-con, provided the clearest evidence to support the proposed hypothesis of the combined risk factor effect of hostility and anger suppression as predictors of PCA. Also in Models 1 and 4, these particular variables showed significant contribution to the variance explained of PCA.

In contrast with the results of previous angiographic studies by Dembroski et al. (11) and Suarez (13), we did not find a significant interaction effect. The impact of CynDis and AX-con on PCA seemed to be additive rather than synergistic, as illustrated in Figure 1. This is also illustrated by the finding that, when interactions were entered in the regression models, the variance explained in any of the models tested was not raised. The somewhat better predictive power of binary models, especially for the CynDis scale, indicates the possibility of a curvilinear association between CynDis and PCA. It is also possible that, despite its high reliability, the relatively short CynDis scale lacks validity over its whole range. These questions, however, need to be studied in larger samples than the present one.

The ANCOVA based on Model 3 showed about two-fold accelerated PCA in the group high in CynDis and high in AX-con, even after we controlled for previously established biological risk factors. This result could be taken as a conservative estimate of the actual impact on PCA by these variables, as indicated by the nontransformed mean values.

In the present study, cynical distrust seemed to be the strongest psychological predictor of PCA. If we accept the argument that the Cook-Medley hostility scale is primarily a measure of cynicism $(20,28)$, our results support and extend the earlier results by Williams et al. (29). On the other hand, in a more recent cross-sectional angiographic study, no association was found between the total Cook-Medley hostility scale and the extent of coronary occlusion (30). Although the sample size in all the studies referred to was about the same, the present study is the only prospective one based on a nonselected population sample. Furthermore, the potential predictive power of the CynDis scale is also supported by a cross-sectional study in which we found this measure to correlate significantly with diastolic blood pressure in men (31). The evidence, as we see it, suggests that CynDis could be the pathogenic element in the Cook-Medley hostility scale. Therefore, the observed inconsistencies in previous results with the total Cook-Medley hostility scale could reflect its nature as a rather multifactorial, nonspecific measure of hostility $(8,20)$.

Correlational analysis revealed that CynDis was only weakly and marginally significantly associated with the other measures of hostility and anger. As indicated by previous research, this particular measure reflects a suspicious and distrustful attitude toward fellow human beings but is not necessarily an indicator of openly aggressive behavior $(20,21)$. 


\section{JULKUNEN et al.}

Thus, a person who scores high on this scale seems to be unable to trust others. Such a person does not expect any unselfish help from others who are seen mostly as dishonest and unreliable. Furthermore, CynDis is apparently a rather stable trait, as indicated by the relatively high test-retest correlation; this observation agrees with previously reported results with the total Cook-Medley hostility scale (28).

The results of the present study indicate that $\mathrm{AX}$ con, especially together with a high level of CynDis, is a risk factor for accelerated PCA. Similar findings were not observed with the measure of AX-in. Thus, individuals with a tendency to extend effort to control angry feelings and behaviors were more at risk than individuals who have a tendency to hold feelings of anger in, pout, withdraw, or harbor grudges. The increased risk associated with the combination of the two scales, CynDis and AX-con, may suggest that it is both the frequency with which the person experiences anger arousal, which may be greater in individuals who are high in cynicism, and a tendency to control the emotion once aroused that has pathogenic effects. The data from this study indicate that there are psychologically subtle but important differences between personality dispositions toward cynicism and distrust, feelings of anger, and anger expression that require more sophisticated and multidimensional measurement. In addition, more research into the complex interactions of various forms of anger, hostility, and anger expression is needed before their health consequences can be better understood.

In summary, the present findings indicated that, in addition to the previously established risk factors (i.e., high serum LDL cholesterol concentration, smoking, and old age), two hostility-related variables (CynDis and AX-con) contributed significantly to the regression equation that explained more than one third of the variation of PCA. The impact of the two psychological variables on PCA seemed to be additive rather than synergistic because interaction effects were negligible.

Because of the relatively small number of subjects in our study, the results must be interpreted cautiously. The relevance of the present findings for the development of clinical manifestations of CHD, the main interest of our prospective study, will be investigated subsequently, based on the whole population sample of the KIHD study.

Parts of this article were presented at the 31st Annual Conference on Cardiovascular Disease Epidemiology of the American Heart Association, Orlando, Florida, 1991, and at the 25th International
Congress of Psychology, Brussels, July 1992. The KIHD study is supported by the Finnish Academy, the Ministry of Education of Finland, and the $\mathrm{Na}$ tional Heart, Lung, and Blood Institute. Preparation of this manuscript was partially supported by a grant from the Signe and Ane Gyllenberg's Foundation to the first author.

\section{REFERENCES}

1. Pickering TG: Should studies of patients undergoing coronary angiography be used to evaluate the role of behavioral risk factors for coronary heart disease? J Behav Med 8:203-211, 1985

2. Salonen JT, Salonen R: Ultrasound B-mode imaging in observational studies in atherosclerosis progression. Circulation 56(suppl):63, 1993

3. Salonen JT, Salonen R: Ultrasonographically assessed carotid morphology and the risk of coronary heart disease. Arterioscler Thromb 11:1245-1249, 1991

4. Williams RB, Barefoot JC: Coronary-prone behavior: The emerging role of the hostility complex. In Houston BK, Snyder CR (eds), Type A Behavior: Research, Theory, and Intervention. New York, Wiley, 1988, 189-211

5. Dembroski TM, Costa PT: Assessment of coronary-prone behavior: A current overview. Ann Behav Med 10:60-63, 1988

6. Matthews KA: Coronary heart disease and type $A$ behaviors: Update on and alternative to the Booth-Kewley and Friedman (1987) quantitative review. Psychol Bull 104:373-380, 1988

7. Goldstein MG, Niaura R: Psychological factors affecting physical condition: Cardiovascular disease literature review. Psychosomatics 33:134-155, 1992

8. Barefoot IC: Developments in the measurement of hostility. In Friedman HS (ed), Hostility, Coping \& Health. Washington, DC, American Psychological Association, 1992, 13-31

9. Spielberger CD, Johnson EH, Russell SF, et al: The experience and expression of anger: Construction and validation of an Anger Expression Scale. In Chesney MA, Rosenman RH (eds), Anger and Hostility in Cardiovascular and Behavioral Disorders. Washington, DC, Hemisphere, 1985, 5-30

10. Smith TW, Frohm KD: What's so unhealthy about hostility? Construct validity and psychosocial correlates of the Cook and Medley Ho scale. Health Psychol 4:503-520. 1985

11. Dembroski TM, MacDougal JM, Williams RB, et al: Components of type A, hostility, and anger-in: Relationship to angiographic findings. Psychosom Med 47:219-233, 1985

12. McDougall JM, Dembroski TM, Dimsdale JE, et al: Components of type A, hostility, and anger-in: Further relationships to angiographic findings. Health Psychol 4:137-152, 1985

13. Suarez EC: The relationship among components of the type A behavior pattern, Cook-Medley hostility scores, and atherosclerosis. Dissertation Abstr Int 47:2635, 1986

14. Salonen JT: Is there a continuing need for longitudinal epidemiologic research-the Kuopio Ischaemic Heart Disease Risk Factor Study. Ann Clin Res 20:46-50, 1988

15. Salonen R, Seppanen K, Rauramaa R, et al: Prevalence of carotid atherosclerosis and serum cholesterol levels in eastern Finland. Arteriosclerosis 8:788-792, 1988

16. Salonen R, Salonen JT: Progression of carotid atherosclerosis and its determinants: A population-based ultrasonography study. Atherosclerosis 81:33-40, 1990 


\section{HOSTILITY AND ATHEROSCLEROSIS}

17. Salonen R, Salonen IT: Intima-media changes in a population study: KIHD. In Boccalon $\mathrm{H}$ (ed), Vascular Medicine. New York, Elsevier Science Publishers, 1993, 301-304

18. Salonen IT, Korpela H, Salonen R, et al: Precision and reproducibility of ultrasonographic measurement of progression of common carotid artery atherosclerosis. Lancet 341:11581159,1993

19. Cook WW, Medley DM: Proposed hostility and pharisaicvirtue scales for the MMPI. J Appl Psychol 38:414-418, 1954

20. Greenglass ER, Julkunen J: Construct validity and sex differences in Cook-Medley hostility. Pers Individ Diff 10:209-218, 1989

21. Greenglass ER, Julkunen J: Cook-Medley hostility, anger, and the type A behavior pattern in Finland. Psychol Rep 68:10591066, 1991

22. Järvikoski A, Härkäpaa K: A brief type-A scale and the occurrence of cardiovascular symptoms. Scand J Rehabil Med 19:115-120, 1987

23. Spielberger CD: State-Trait Anger Inventory, Research Edition, Professional Manual. Odessa, FL: Psychologic Assessment Resources, 1986

24. Romanov K, Koskenvuo M, Kaprio J: Components of aggressiveness (in Finnish). Sosiaalilaaketieteellinen Aikakauslehti/J Soc Med 28:308-313, 1991

25. Kaplan GA, Salonen IT: Socioeconomic conditions in child- hood and ischaemic heart disease during middle age. BM] 301:1121-1123, 1990

26. Salonen JT, Salonen R, Seppänen K, et al: Interactions of serum copper, selenium, and low density lipoprotein cholesterol in atherogenesis. BMJ 302:756-760, 1991

27. User's Guide: SPSS-X. A Complete Guide to SPSS-X Language and Operations, 3rd Edition. New York, McGraw-Hill, 1988

28. Williams RB: Type A behavior and coronary heart disease: Something old, something new. Behav Med Update 6:29-33, 1984

29. Williams RB, Haney TL, Lee KL, et al: Type A behavior, hostility, and coronary atherosclerosis. Psychosom Med 42:539-549, 1980

30. Helmer DC, Ragland DR, Syme SL: Hostility and coronary artery disease. Am J Epidemiol 133:112-122, 1991

31. Julkunen J, Koshonen HJ, Lahti T: Work stress, anger, and blood pressure in North Karelian men and women. In Motoaki $\mathrm{H}$, Misumi J, Wilpert B (eds), Proceedings of the 22nd International Congress of Applied Psychology, Vol. 3. Hillsdale, N), Lawrence Erlbaum Associates, 1992, 359-362

32. Spielberger CD, Krasner SS, Solomon EP: The experience, expression, and control of anger. In Janisse MP (ed), Health Psychology: Individual Differences and Stress. New York, Springer Verlag, 1988, 89-108 